\title{
Intervenção Coronária Percutânea em Pontes de Veia Safena com Uso de Stents Farmacológicos: Resultados Agudos e Tardios dos Pacientes Incluídos no Registro DESIRE
}

\author{
Jose de Ribamar Costa Jr. ${ }^{1}$, Amanda G. M. R. Sousa', Adriana Moreira1', Ricardo Costa', Galo Maldonado',

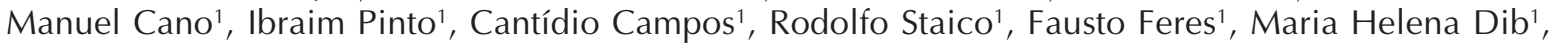 \\ Ricardo Pavanello', J. Eduardo Sousa ${ }^{1}$
}

\section{RESUMO}

Introdução: Mesmo com o advento dos stents farmacológicos, a intervenção coronária percutânea em pontes de veia safena permanece desafiadora, com elevada incidência de complicações agudas e incerteza dos resultados tardios. Neste estudo, buscamos avaliar os resultados clínicos de pacientes com lesões em pontes de veia safena tratados com stents farmacológicos. Método: Entre maio de 2002 e janeiro de 2009, 151 pacientes com 196 lesões em pontes de veia safena foram consecutivamente submetidos a intervenção coronária percutânea com 211 stents farmacológicos e incluídos neste estudo. Os pacientes foram pré-tratados com enoxaparina, clopidogrel e ácido acetilsalicílico por 3 a 5 dias antes da intervenção coronária percutânea. A terapia antiplaquetária dupla (ácido acetilsalicílico + clopidogrel) foi prescrita por 12 meses. Objetivou-se determinar as taxas de eventos cardíacos adversos maiores (ECAM) no período hospitalar e na fase tardia. Seguimento clínico foi obtido com 1, 6 e 12 meses e, então, anualmente. Resultados: A média de idade da população avaliada foi de 68,2 anos, com predominância do sexo masculino $(89,1 \%)$. Diabetes melito foi observado em $30,7 \%$ dos casos e a média de idade dos enxertos venosos foi de 10,4 anos. Sucesso angiográfico foi obtido em $98,2 \%$ dos casos. Na fase hospitalar, a taxa de ECAM foi de $8,7 \%$, sobretudo à custa de elevação enzimática pós-procedimento $(7,1 \%)$. No primeiro ano de evolução, a taxa de ECAM elevou-se para $14,2 \%$, com $3,9 \%$ de nova revascularização da lesãoalvo. No seguimento mais tardio (média de 2,6 anos), a taxa de ECAM atingiu 28,5\%, com $6 \%$ de óbito cardíaco e $16,6 \%$ de infarto agudo do miocárdio. Nessa população, foram observados 5 casos de trombose (todos definitivos). Conclusão: Na presente subanálise do Registro DESIRE, o

\section{ABSTRACT}

\section{Percutaneous Coronary Intervention in Saphenous Vein Bypass Grafts with Drug-Eluting Stents: Early and Late Outcomes of Patients in the DESIRE Registry}

Introduction: Despite the advent of drug-eluting stents, percutaneous coronary interventions in saphenous vein bypass grafts remain a challenge, with a high incidence of early complications and uncertainty regarding late outcomes. This study was aimed at evaluating clinical outcomes of patients with saphenous vein graft lesions treated with drug-eluting stents. Method: From May 2002 to January 2009, a total of 151 patients with 196 saphenous vein graft lesions were consecutively submitted to percutaneous coronary intervention with 211 drug-eluting stents and included in this trial. Patients were pretreated with enoxiparin, clopidogrel and acetylsalicylic acid for 3 to 5 days prior to percutaneous coronary intervention. Dual antiplatelet therapy (acetylsalicylic acid + clopidogrel) was prescribed for 12 months. The objective was to determine the rate of major adverse cardiac events (MACE) during hospitalization and in the long-term. Clinical follow-up was obtained at 1, 6 and 12 months and then, yearly after that. Results: Mean age was 68.2 years, with a prevalence of male patients $(89.1 \%)$. Diabetes mellitus was observed in $30.7 \%$ cases and mean age of venous grafts was 10.4 years. Angiographic success was obtained in $98.2 \%$ of the cases. During hospitalization, MACE rate was $8.7 \%$, especially due to post-procedural enzyme increase $(7.1 \%)$. In the first follow-up year, the rate of MACE increased to $14.2 \%$, with a $3.9 \%$ rate of target-lesion revascularizations. In the late follow-up (mean of 2.6 years), the rate of MACE increased to $28.5 \%$, with $6.0 \%$ of cardiac deaths and $16.6 \%$

\footnotetext{
1 Instituto de Ensino e Pesquisa - Hospital do Coração - Associação do Sanatório Sírio - São Paulo, SP, Brasil.

Correspondência: J. Eduardo Sousa. Rua Desembargador Eliseu Guilherme, 147 - Paraíso - São Paulo, SP, Brasil - CEP 04004-030 E-mail: jesousa@uol.com.br

Recebido em: 8/2/2009 • Aceito em: 8/6/2009
} 
Costa Jr. JR, et al. Intervenção Coronária Percutânea em Pontes de Veia Safena com Uso de Stents Farmacológicos: Resultados Agudos e Tardios dos Pacientes Incluídos no Registro DESIRE. Rev Bras Cardiol Invas. 2009;17(2):202-8.

uso da terapêutica antitrombótica otimizada (ácido acetilsalicílico + tienopiridínico + heparina de baixo peso, por 3 a 5 dias pré-intervenção) e o uso de stents farmacológicos para o tratamento de pontes de veia safena correlacionaramse com excelentes desfechos clínicos imediatos e no primeiro ano pós-intervenção percutânea. No entanto, após o primeiro ano, tais resultados sofrem marcante deterioração, refletindo o caráter mais agressivo da doença vascular nos condutos venosos. As taxas de trombose podem ser consideradas baixas, favorecendo a segurança desses instrumentais em tão complexo cenário.

DESCRITORES: Angioplastia transluminal percutânea coronária. Stents farmacológicos. Veia safena.
$\mathbf{T}$ radicionalmente, a intervenção coronária em pontes de veia safena tem sido considerada de alto risco, dada a incidência mais elevada de eventos adversos, tanto na fase hospitalar como no seguimento mais tardio.

Dentre os possíveis fatores relacionados a esses piores resultados, destacam-se: 1) pacientes em geral mais graves, frequentemente com histórico de múltiplos procedimentos de revascularização prévios, presença de disfunção ventricular esquerda e acometimento multiarterial; 2) lesões com características angiográficas de maior complexidade, com presença não infrequente de trombo, úlcera e degeneração do conduto venoso; e 3) história natural da doença aterosclerótica diferente dos vasos nativos, com progressão da doença mais acelerada e reestenose mais frequente em comparação às artérias coronárias nativas ${ }^{1-3}$.

Nesse contexto, a combinação de regime antitrombótico otimizado pré-intervenção e uso de stents farmacológicos durante a angioplastia coronária poderia reduzir os desfechos adversos, tanto na fase aguda como no seguimento a longo prazo.

Na presente análise, objetivamos avaliar a eficácia dessa combinação em pacientes consecutivos, nãoselecionados, tratados exclusivamente com stents farmacológicos em um único centro em São Paulo.

\section{MÉTODO}

\section{Casuística de desenho do estudo}

Desde maio de 2002, quando o primeiro stent farmacológico foi aprovado para uso clínico no Brasil, todos os pacientes tratados exclusivamente com esses instrumentais no Hospital do Coração - Associação do Sanatório Sírio, em São Paulo, foram incluídos no Drug-Eluting Stents In the Real World Registry (Registro DESIRE). Os resultados gerais do DESIRE já foram previamente publicados, razão pela qual não nos deteremos em pormenores do registro ${ }^{4,5}$. of acute myocardial infarctions. Five cases of thrombosis (all of them definitive) were observed in this population. Conclusion: In the present subanalysis of the DESIRE Registry, the use of optimized antithrombotic therapy (acetylsalicylic acid + thienopyridines + low molecular weight heparin, for 3 to 5 days before the intervention) and the use of drug-eluting stents for the treatment of saphenous vein grafts is correlated to excellent early clinical outcomes and in the first year after percutaneous intervention. However, after the first year, these results significantly deteriorate, indicating the more aggressive character of vascular disease in the venous conduits. Thrombosis rates may be considered low, favoring the safety of these devices in such complex scenario.

DESCRIPTORS: Angioplasty, transluminal, percutaneous coronary. Drug-eluting stents. Saphenous vein.

O presente estudo representa uma subanálise daquele registro, envolvendo todos os pacientes que apresentavam como lesão-alvo obstrução significativa localizada em pontes de veia safena, tratados até janeiro de 2009. Foram excluídos pacientes tratados com stents farmacológicos e não-farmacológicos no mesmo procedimento, aqueles com contraindicação a terapêutica antitrombótica otimizada utilizada em nossa Instituição nesse cenário (descrição a seguir), e aqueles pacientes tratados em fase aguda de infarto agudo do miocárdio com supradesnivelamento do segmento ST.

A obstrução significativa foi definida pela ocorrência de sintomas isquêmicos e/ou exame não-invasivo indicativo de isquemia no território tratado, na presença de obstrução $\geq 50 \%$, à angiografia (avaliação visual) no vaso-alvo.

\section{Regime antitrombótico}

O protocolo antitrombótico consistiu na administração de dois agentes antiplaquetários: ácido acetilsalićlico (100-200 mg) iniciado pelo menos 72 horas antes do procedimento e prescrito indefinidamente; e clopidogrel (dose de ataque de 300-600 mg) também iniciado 72 horas antes da intervenção e mantido por um período mínimo de 12 meses. Adicionalmente, os pacientes receberam dose plena de heparina de baixo peso molecular (enoxaparina na dose de $1 \mathrm{mg} / \mathrm{kg}$ a cada 12 horas), nos 3 a 5 dias que antecederam a intervenção.

Durante o procedimento, manteve-se o uso de heparina de baixo peso molecular. A decisão sobre a utilização de inibidores da glicoproteína Ilb/IIla ficou a critério do operador.

\section{Intervenção coronária percutânea}

O implante do stent farmacológico seguiu a técnica contemporânea estabelecida, com o cuidado de, 
Costa Jr. JR, et al. Intervenção Coronária Percutânea em Pontes de Veia Safena com Uso de Stents Farmacológicos: Resultados Agudos e Tardios dos Pacientes Incluídos no Registro DESIRE. Rev Bras Cardiol Invas. 2009;17(2):202-8.

sempre que possível, realizar o implante direto do stent. Quando necessária, a pré-dilatação deveria ser realizada com balões curtos insuflados a baixas pressões, visando a evitar injúria nos segmentos adjacentes à lesão. O stent deveria ser implantado de forma a garantir a completa cobertura da lesão e, quando necessário, mais de um stent poderia ser empregado com a sobreposição de suas bordas. O uso de filtro de proteção distal ficou a critério do operador.

Após o implante de stent, a pós-dilatação, quando indicada, deveria ser realizada com balões com extensão menor que a do stent implantado, com o cuidado de não ultrapassar suas bordas, evitando mais uma vez a injúria nos segmentos não cobertos pela prótese. Utilizamos os seguintes stents farmacológicos: stent Cypher ${ }^{\mathrm{TM}}$ (Cordis, Johnson \& Johnson, Miami Lakes, Estados Unidos), com eluição de sirolimus; stent Taxus $^{\mathrm{TM}}$ (Boston Scientific Corporation, Natick, Estados Unidos), com eluição de paclitaxel; e Endeavor ${ }^{\mathrm{TM}}$ (Medtronic, Santa Rosa, Estados Unidos), com eluição de zotarolimus. Esses stents estavam disponíveis em variações de extensão de $8 \mathrm{~mm}$ a $33 \mathrm{~mm}$ e diâmetros de 2,25 $\mathrm{mm}$ a $3,5 \mathrm{~mm}$ para o stent Cypher ${ }^{\mathrm{TM}}$, extensões de $8 \mathrm{~mm}$ a $32 \mathrm{~mm}$ e diâmetros de 2,25 $\mathrm{mm}$ a 4,5 mm para o stent Taxus ${ }^{\mathrm{TM}}$, e extensões de $8 \mathrm{~mm}$ a $30 \mathrm{~mm}$ e diâmetros de 2,5 $\mathrm{mm}$ a $4 \mathrm{~mm}$ para Endeavor ${ }^{\mathrm{TM}}$. A escolha do tipo de stent farmacológico ficou a critério de cada operador.

A angiografia coronária quantitativa foi realizada imediatamente antes e após o implante do stent, por técnico treinado e experiente. A análise do segmento tratado compreendeu a área coberta pelo stent e as bordas $(5 \mathrm{~mm})$ proximal e distal. As mensurações angiográficas incluíram as referências proximal, distal e interpolada, o diâmetro mínimo da luz, a porcentagem de obstrução e a extensão da lesão. Além disso, o ganho imediato foi avaliado a partir do cálculo da diferença entre o diâmetro mínimo da luz aferido imediatamente após e antes do implante do stent. Durante a hospitalização, todos os pacientes foram submetidos a avaliação com eletrocardiograma e marcadores bioquímicos - creatina quinase fração $\mathrm{MB}$ (CK-MB) -, em três momentos: antes e imediatamente após a intervenção, e na alta hospitalar.

\section{Objetivos do estudo e definições}

O desfecho primário deste estudo foi a avaliação da ocorrência de eventos cardíacos maiores na fase intra-hospitalar e no seguimento clínico a longo prazo. Os desfechos secundários incluíram as taxas de revascularização da lesão-alvo e de trombose do stent em todo o período do seguimento.

Os eventos cardíacos maiores foram definidos como óbito cardíaco, infarto do miocárdio e revascularização da lesão-alvo. Os óbitos foram classificados como cardíacos e não-cardíacos. Óbitos de causas indeterminadas foram relatados como cardíacos. Os infartos do miocárdio foram definidos como o aparecimento de novas ondas $Q$ em duas derivações contíguas do eletrocardiograma e/ou a elevação da CK-MB > 3 vezes o limite superior do normal. Todas as reintervenções, percutâneas ou cirúrgicas, resultantes da recorrência da obstrução (> 50\%) dentro do stent implantado no procedimento-índice ou no segmento que incluía os $5 \mathrm{~mm}$ proximais e os $5 \mathrm{~mm}$ distais ao stent foram classificadas como revascularização da lesão-alvo. A classificação proposta pelo Academic Research Consortium foi utilizada para a definição da trombose do stent como definitiva (confirmação angiográfica ou anatomopatológica), provável (morte súbita $\leq 30$ dias pós-implante de stents farmacológicos ou infarto relacionado à região da artéria tratada, mesmo sem confirmação angiográfica) e possível (morte súbita > 30 dias pós-stents farmacológicos). Quanto à distribuição temporal, as tromboses protéticas ainda foram divididas em: agudas e subagudas ( $\leq 30$ dias), tardias (31 dias a 360 dias) e muito tardias (> 360 dias). O sucesso angiográfico foi definido pela presença de lesão residual $<20 \%$ associada à presença de fluxo coronário TIMI 3 na ausência de trombos e/ou dissecções. O sucesso do procedimento foi considerado como o sucesso angiográfico na ausência de eventos cardíacos maiores.

A função ventricular esquerda foi avaliada pelo cálculo da fração de ejeção (\% FE) e classificada como: normal (\% FE $\geq 55 \%$ ), disfunção discreta (\% FE $\geq 40 \%$ $<55 \%$ ), disfunção moderada ( $\% \mathrm{FE} \geq 30 \%<40 \%$ ), e disfunção significativa (\% FE < 30\%).

A insuficiência renal crônica foi definida pela presença de valores séricos da creatinina $\geq 1,5 \mathrm{mg} / \mathrm{dl}$ e pela taxa de filtração glomerular (clearance) $<60 \mathrm{ml} /$ $\min / 1,73 \mathrm{~m}^{2}$.

O seguimento clínico foi obtido por visita médica ou por telefone, seguindo protocolo pré-definido, ao final de 30 dias, seis meses e um ano. A partir de então, o seguimento foi realizado anualmente.

\section{Análise estatística}

As variáveis categóricas estão descritas como frequências e porcentagens e comparadas pelo teste de qui-quadrado. As variáveis contínuas estão descritas como média e desvio padrão e comparadas pelo teste $t$ de Student. A sobrevida livre de eventos cardíacos maiores foi estimada pela curva de KaplanMeier. Na presente análise, o valor de $\mathrm{P}<0,05$ foi considerado significativo. Utilizou-se o pacote estatístico SPSS (versão 13.0) para realizar esta análise.

\section{RESULTADOS}

Entre maio de 2002 e janeiro de 2009, 151 pacientes portadores de lesões em pontes de veia safena foram identificados (do total de 2.854 pacientes do DESIRE - 5,3\%). 
Costa Jr. JR, et al. Intervenção Coronária Percutânea em Pontes de Veia Safena com Uso de Stents Farmacológicos: Resultados Agudos e Tardios dos Pacientes Incluídos no Registro DESIRE. Rev Bras Cardiol Invas. 2009;17(2):202-8.

A Tabela 1 contém as principais características dos pacientes incluídos neste estudo. A maioria era do sexo masculino $(81,9 \%)$, com média de idade de $68,2 \pm 10$ anos. Diabetes melito foi observado em $30,7 \%$ dessa coorte. Praticamente $30 \%$ da população apresentava quadro clínico inicial de angina instável e a média de idade dos enxertos venosos tratados era de 10,4 $\pm 2,7$ anos. Presença de disfunção de ventrículo esquerdo considerada moderada/grave foi de $34,5 \%$.

O território mais frequentemente revascularizado foi o da artéria descendente anterior, sendo a presença de trombo detectada em $6,1 \%$ dos casos. A angiografia quantitativa pré-intervenção demonstrou que o diâmetro de referência e a extensão das lesões tratadas eram de 3,1 $\pm 0,6 \mathrm{~mm}$ e $14,6 \pm 6,3 \mathrm{~mm}$, respectivamente. Após a intervenção, a lesão residual foi de $5,2 \pm 3,5 \%$. Stent Cypher ${ }^{\text {TM }}$ foi utilizado na maioria dos casos $(75,9 \%)$ e os filtros de proteção distal foram utilizados em menos de $10 \%$ dos casos. As taxas de fluxo coronário TIMI III e de sucesso angiográfico foram, respectivamente, de 99,4\% e 98,6\%. A Tabela 2 detalha o procedimento e apresenta os resultados da angiografia coronária quantitativa pré e pós-intervenção.

O seguimento clínico tardio $(2,6 \pm 1,2$ anos $)$ foi obtido em 98,3\% dos pacientes. Na fase hospitalar, observou-se taxa combinada de eventos maiores de 8,7\%, sobretudo elevação enzimática pós-procedimento

TABELA 1

Características clínicas e angiográficas de base

\begin{tabular}{lc}
\hline & $\begin{array}{c}\text { 151 pacientes } \\
\text { (196 lesões) }\end{array}$ \\
\hline Média de idade, anos & $68,2 \pm 10$ \\
Sexo feminino, \% & 18,1 \\
Diabetes melito, \% & 30,7 \\
HAS, \% & 82,7 \\
Dislipidemia, \% & 70,9 \\
Tabagismo atual, \% & 7,1 \\
História familiar de DAC, \% & 40,9 \\
Angioplastia coronária prévia, \% & 27,6 \\
Apresentação clínica & 70,1 \\
Isquemia silenciosa/angina estável, \% & 29,9 \\
Angina instável/IAM sem supra de ST, \% & \\
Território tratado & 33,6 \\
Artéria descendente anterior, \% & 36,4 \\
Artéria circunflexa, \% & 30 \\
Artéria coronária direita, \% & 34,5 \\
Disfunção de VE (FE < 40\%), \% & 10,3 \\
> de 1 território tratado, \% & \\
\hline DAC = doença arterial coronária; FE = fração de ejeção; \\
HAS = hipertensão arterial sistêmica; IAM = infarto agudo \\
do miocárdio; VE = ventrículo esquerdo. \\
\hline
\end{tabular}

(infarto agudo do miocárdio sem supradesnivelamento do segmento $\mathrm{ST}=7,1 \%$ ). No seguimento até um ano, a taxa de eventos combinados elevou-se para $14,2 \%$, com $1,6 \%$ de óbito cardíaco e 3,9\% de nova revascularização da lesão-alvo. No entanto, chama atenção a rápida deterioração dos resultados após o primeiro ano de evolução, confirmando o caráter mais agressivo da degeneração dos enxertos venosos. Apenas $71,5 \%$ dos pacientes estavam livres de eventos cardíacos adversos maiores a longo prazo (Figura 1), com $6 \%$ de óbito cardíaco e $16,6 \%$ de infarto do miocárdio (Tabela 3). A taxa de trombose de stent nessa população foi de $3,3 \%$, sendo todos os casos $(n=5)$ classificados como definitivos. Quanto à distribuição temporal, duas tromboses ocorreram no primeiro ano do procedimento (ambas tardias), enquanto as outras três foram classificadas como muito tardias (> 1 ano).

Por fim, vale ressaltar que, na análise da população global incluída no Registro DESIRE, o tratamento de pontes de veia safena representou preditor independente de eventos cardíacos adversos maiores (risco relativo $[R R]=1,68$, intervalo de confiança de $95 \%$ [IC 95\%] 1,22-2,18; P = 0,001) e de nova revascularização da lesão-alvo ( $R R=4,5$, IC 95\% 1,7-7,1; $P<0,001)$.

TABELA 2 Análise angiográfica quantitativa

\begin{tabular}{lc}
\hline Características & $\begin{array}{c}\mathbf{n}=\mathbf{1 9 6} \text { lesões/ } \\
\mathbf{2 1 1} \text { stents }\end{array}$ \\
\hline Extensão da lesão, mm & $14,6 \pm 6,3$ \\
Extensão do stent, mm & $18,7 \pm 4,2$ \\
Uso de dispositivos de proteção, \% & 5,6 \\
Stent implantado, \% & \\
Cypher $^{\top M}$ & 75,9 \\
Taxus $^{\text {TM }}$ & 18,9 \\
Endeavor & \\
Pré-dilatação, \% & 5,2 \\
Pós-dilatação, \% & 24,8 \\
Extensão do stent/extensão da lesão, mm & $1,28 \pm 0,3$ \\
Pressão máxima de liberação do stent, atm & $15,8 \pm 3,4$ \\
Diâmetro médio de referência do vaso, mm & $3,1 \pm 0,6$ \\
Diâmetro luminal mínimo, mm & \\
Pré-procedimento & $1 \pm 0,6$ \\
Pós-procedimento & $3,1 \pm 0,5$ \\
Diâmetro de estenose, \% & \\
Pré-procedimento & \\
Pós-procedimento & $67,6 \pm 9,1$ \\
Ganho agudo, mm & $5,2 \pm 3,5$ \\
Fluxo TIMl III, \% & $2,1 \pm 0,5$ \\
Sucesso angiográfico, \% & 99,4 \\
Sucesso do procedimento, \% & 98,6 \\
& 92,9 \\
\hline
\end{tabular}




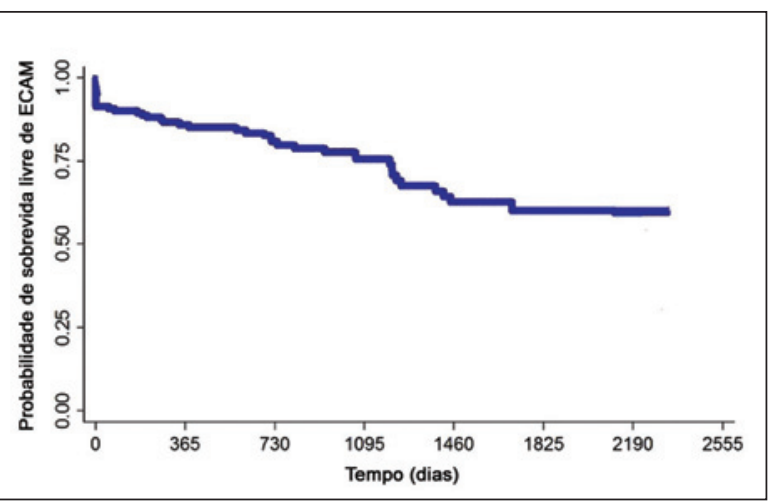

Figura 1 - Curva de sobrevida livre de eventos cardíacos adversos maiores (ECAM)

TABELA 3

\section{Eventos cardíacos maiores nas fases hospitalar e tardia}

\begin{tabular}{lc}
\hline Eventos & $\mathbf{n}=\mathbf{1 5 1}$ pacientes \\
\hline Fase hospitalar & \\
$\quad$ Óbito cardíaco, \% & 0,8 \\
IAM, \% & $7,1^{*}$ \\
RLA, \% & 0,8 \\
Até 1 ano de seguimento & \\
Óbito cardíaco, \% & 1,6 \\
IAM, \% & 8,7 \\
RLA, \% & 3,9 \\
Seguimento tardio (2,6 $\pm 1,2$ anos) & 6 \\
Óbito cardíaco, \% & 16,6 \\
IAM, \% & 9,9 \\
RLA, \% & \\
\hline Todos foram IAM sem supradesnivelamento do segmento ST. \\
IAM = infarto agudo do miocárdio; RLA = revascularização \\
da lesão-alvo. \\
\hline
\end{tabular}

\section{DISCUSSÃo}

São dois os principais achados da presente análise: 1) o uso de stents farmacológicos mostrou-se bastante seguro nesta experiência de mundo real, com baixas taxas de trombose em seguimento bastante prolongado; e 2) apesar da eficácia desses novos dispositivos em reduzir as taxas de nova intervenção da lesão-alvo no primeiro ano após seu implante, a longo prazo esses resultados são em parte perdidos, provavelmente em decorrência do caráter mais agressivo de progressão da doença nos condutos venosos.

Ainda que os stents farmacológicos tenham demonstrado marcante redução das taxas de reestenose nos mais variados e complexos cenários clínicos e angiográficos, a relativa escassez de dados sobre a segurança e a eficácia tardia desses instrumentais no tratamento de lesões em pontes de veia safena fez com que, nos primeiros anos após sua introdução no cenário clínico, seu uso fosse considerado off-label e mesmo proscrito em alguns centros.

Tal cenário tem se modificado recentemente com a publicação dos primeiros estudos comparativos, em pontes de veia safena, entre esses novos dispositivos e os stents não-farmacológicos, ainda que haja alguma inconsistência entre os dados publicados.

Em 2006, Vermeersch et al. ${ }^{6}$ publicaram os resultados iniciais do primeiro estudo randomizado comparando um stent farmacológico (Cypher ${ }^{\mathrm{TM}}$ ) a seu correspondente sem fármaco (Bx Velocity ${ }^{\mathrm{TM}}$ ), no cenário da intervenção em pontes de veia safena. Esses pesquisadores observaram que, no primeiro ano após a intervenção, o uso de stents farmacológicos associou-se a significativa redução da taxa de revascularização da lesão-alvo em comparação à coorte tratada com stents não-farmacológicos (5,3\% vs. 21,6\%; $R R=0,24$, IC 95\% 0,05-1,0; P $<0,047)$, com semelhantes taxas de óbito e infarto. No que tange aos resultados angiográficos e ultrassonográficos, o uso de stents farmacológicos também se associou a marcante redução, tanto na perda tardia quanto na porcentagem de obstrução do stent, sem que houvesse maiores preocupações naquela ocasião quanto à má-aposição tardia e a outros sinais indiretos de possível toxicidade local induzida pelo fármaco antiproliferativo ${ }^{7}$. Entretanto, o mesmo grupo decidiu seguir esses pacientes por um período de até três anos após o implante, e, paradoxalmente, após o primeiro ano, foi observada significativa diferença $(29 \%)$ na taxa de óbito entre os dois grupos, desfavorecendo aqueles pacientes tratados com stents farmacológicos (IC 95\% 14-45; $\mathrm{P}<0,001$ ), o que foi atribuído à possível maior ocorrência de trombose tardia entre aqueles pacientes ${ }^{8}$. Contra esses achados pesa o fato de que as populações em ambos os braços eram muito pequenas (38 pacientes no grupo stent farmacológico e 37 no grupo stent não-farmacológico), não havendo poder estatístico para identificar diferenças em desfechos clínicos, podendo essa diferença ser mero "achado" estatístico.

Em 2008, Assali et al. ${ }^{9}$ publicaram os resultados da comparação entre 68 pacientes tratados com stents farmacológicos e 43 pacientes que receberam stents não-farmacológicos para lesões em pontes de veia safena. Nesse estudo, com dois anos de seguimento clínico, o uso de stents não-farmacológicos associou-se a marcante redução da sobrevida livre de eventos (58,1\% no grupo stents não-farmacológicos vs. 79,4\% no grupo stents farmacológicos; $P=0,02)$, essencialmente em decorrência da redução da taxa de nova revascularização da lesão-alvo (14,7\% vs. 32,6\%; $P=0,03)$. Notavelmente não se observou trombose após o primeiro ano entre aqueles pacientes.

Ainda em 2008, van Twisk et al. ${ }^{10}$ apresentaram os resultados de quatro anos da análise combinada 
Costa Jr. JR, et al. Intervenção Coronária Percutânea em Pontes de Veia Safena com Uso de Stents Farmacológicos: Resultados Agudos e Tardios dos Pacientes Incluídos no Registro DESIRE. Rev Bras Cardiol Invas. 2009;17(2):202-8.

dos registros Rapamycin Eluting Stent Evaluated at Rotterdam Cardiology Hospital (RESEARCH) e TaxusStent Evaluated At Rotterdam Cardiology Hospital (TSEARCH), comparando Cypher ${ }^{\mathrm{TM}}$ e Taxus ${ }^{\mathrm{TM}}$ aos stents não-farmacológicos para o tratamento de pontes de veia safena. Ao final do seguimento, 61,5\% dos pacientes tratados com stents farmacológicos e 46,8\% daqueles que receberam stents não-farmacológicos $(R R=0,77, I C 95 \% 0,51-1,16)$ estavam livres de qualquer evento cardíaco maior. No que se refere à nova revascularização da lesão-alvo, houve tendência a menor necessidade de nova revascularização entre os indivíduos que receberam stents farmacológicos $(81,6 \%$ vs. $69 \%$; RR =0,53, IC 95\% 0,27-1,05), sem que houvesse diferença nas taxas de infarto agudo do miocárdio e óbito.

Finalmente, em 2009, Brilakis et al. ${ }^{11}$ publicaram os resultados do estudo randomizado Stenting of Saphenous Vein Grafts (SOS), comparando stents farmacológicos Taxus ${ }^{\mathrm{TM}}$ a stents não-farmacológicos no tratamento de pontes de veia safena (39 pacientes no grupo stents não-farmacológicos e 41 pacientes no grupo stents farmacológicos). No período de seguimento clínico reportado (média de 1,5 ano), o uso de stents farmacológicos associou-se a significativa redução da taxa de eventos cardíacos maiores combinados (28\% vs. 5\%; RR =0,38, IC 95\% 0,15-0,74; P < 0,003), bem como a falência do vaso-alvo ( $46 \%$ vs. $22 \%$; RR = 0,65; IC 95\% 0,42-0,96; $\mathrm{P}<0,03)$, sem que houvesse diferença nas taxas de infarto e óbito.

Ainda que nosso Registro não possua um grupo controle tratado com stents não-farmacológicos, tratase de uma das maiores experiências publicadas com stents farmacológicos em pontes de veia safena, e os resultados apresentados reproduzem a maioria dos braços tratados com stents farmacológicos nos estudos mencionados. Entretanto, como já salientado, o desfecho tardio das pontes de veia safena é notavelmente inferior ao reportado em outros cenários, pela gravidade do paciente e pela rápida progressão da doença nos condutos venosos.

\section{Limitações}

A principal limitação deste estudo refere-se à ausência de um grupo controle tratado com stents nãofarmacológicos. Deve-se, também, ressaltar a predominância de um tipo de stent farmacológico (Cypher ${ }^{\mathrm{TM}}$ ) sobre os demais, impossibilitando comparar o desempenho dos diferentes stents farmacológicos disponíveis. Por último, o uso de terapêutica adjunta antitrombótica otimizada (ácido acetilsalicílico + tienopiridínico + heparina de baixo peso molecular) por 3 a 5 dias pré-intervenção não foi comparado ao tratamento padrão antiplaquetário (ácido acetilsalicílico + tienopiridínico) preconizado pelas diretrizes, não nos permitindo concluir se uma abordagem é superior à outra.

\section{CONCLUSÕES}

Na presente subanálise do Registro DESIRE, o uso da terapêutica antitrombótica otimizada (ácido acetilsalicílico + tienopiridínico + heparina de baixo peso, por 3 a 5 dias pré-intervenção) e o uso de stents farmacológicos para o tratamento de pontes de veia safena correlacionaram-se com excelentes desfechos clínicos imediatos e no primeiro ano pós-intervenção percutânea. No entanto, após o primeiro ano, tais resultados sofrem marcante deterioração, refletindo o caráter mais agressivo da degeneração dos condutos venosos. As taxas de trombose podem ser consideradas baixas, favorecendo a segurança desses instrumentais em tão complexo cenário.

Finalmente, é nossa opinião que esses novos instrumentais devam ser rotineiramente incorporados à prática clínica no tratamento percutâneo de pontes de veia safena, uma vez que não acrescentam riscos em relação aos stents não-farmacológicos, e, por outro lado, marcadamente reduzem a necessidade de novos procedimentos entre esses complexos pacientes muitas vezes com múltiplas intervenções prévias.

\section{CONFLITO DE INTERESSES}

Os autores declararam inexistência de conflito de interesses.

\section{REFERÊNCIAS BIBLIOGRÁFICAS}

1. van Beusekom HM, van der Giessen WJ, van Suylen R, Bos E, Bosman FT, Serruys PW. Histology after stenting of human saphenous vein bypass grafts: observations from surgically excised grafts 3 to 320 days after stent implantation. J Am Coll Cardiol. 1993;21(1):45-54.

2. Depre C, Havaux X, Wijns W. Pathology of restenosis in saphenous bypass grafts after long-term stent implantation. Am J Clin Pathol. 1998;110(3):378-84.

3. Ribichini F, Pugno F, Ferrero V, Wijns W, Vacca G, Vassanelli $C$, et al. Long-term histological and immunohistochemical findings in human venous aorto-coronary bypass grafts. Clin Sci (Lond). 2008;114(3):211-20.

4. Sousa AGMR, Costa Jr. JR, Moreira A, Costa RA, Cano MN, Andrade GAM, et al. Evolução clínica tardia dos stents farmacológicos: segurança e eficácia até cinco anos do registro DESIRE. Rev Bras Cardiol Invas. 2007;15(3):221-7.

5. Sousa A, Costa Jr. JR, Moreira AC, Cano M, Maldonado G, Costa RA, et al.; Drug-Eluting Stents in the Real World (DESIRE) Registry. Long-term clinical outcomes of the DrugEluting Stents in the Real World (DESIRE) Registry. J Interv Cardiol. 2008;21(4):307-14.

6. Vermeersch $\mathrm{P}$, Agostoni $\mathrm{P}$, Verheye $\mathrm{S}$, Van den Heuvel P, Convens C, Bruining N, et al. Randomized double-blind comparison of sirolimus-eluting stent versus bare-metal stent implantation in diseased saphenous vein grafts: sixmonth angiographic, intravascular ultrasound, and clinical follow-up of the RRISC Trial. J Am Coll Cardiol. 2006;48(12): 2423-31.

7. Agostoni $\mathrm{P}$, Vermeersch $\mathrm{P}$, Semeraro $\mathrm{O}$, Verheye $\mathrm{S}$, Van Langenhove G, Van den Heuvel $P$, et al. Intravascular ultrasound comparison of sirolimus-eluting stent versus bare metal stent implantation in diseased saphenous vein grafts 
Costa Jr. JR, et al. Intervenção Coronária Percutânea em Pontes de Veia Safena com Uso de Stents Farmacológicos: Resultados Agudos e Tardios dos Pacientes Incluídos no Registro DESIRE. Rev Bras Cardiol Invas. 2009;17(2):202-8.

(from the RRISC [Reduction of Restenosis In Saphenous Vein Grafts With Cypher Sirolimus-Eluting Stent] trial). Am J Cardiol. 2007;100(1):52-8.

8. Vermeersch P, Agostoni P, Verheye S, Van den Heuvel P, Convens C, Van den Branden F, et al.; DELAYED RRISC (Death and Events at Long-term follow-up AnalYsis: Extended Duration of the Reduction of Restenosis In Saphenous vein grafts with Cypher stent) Investigators. Increased late mortality after sirolimus-eluting stents versus bare-metal stents in diseased saphenous vein grafts: results from the randomized DELAYED RRISC Trial. J Am Coll Cardiol. 2007;50(3):261-7.

9. Assali A, Raz Y, Vaknin-Assa H, Ben-Dor I, Brosh D,
Teplitsky I, et al. Beneficial 2-years results of drug-eluting stents in saphenous vein graft lesions. Eurolntervention. 2008;4(1):108-14.

10. van Twisk PH, Daemen J, Kukreja N, van Domburg RT, Serruys PW. Four-year safety and efficacy of the unrestricted use of sirolimus- and paclitaxel-eluting stents in coronary artery bypass grafts. Eurolntervention. 2008;4(3):311-7.

11. Brilakis ES, Lichtenwalter C, Lemos JA, Roesle M, Obel O, Haagen D, et al. A randomized controlled trial of a paclitaxeleluting stent versus a similar bare-metal stent in saphenous vein graft lesions the SOS (Stenting of Saphenous Vein Grafts) trial. J Am Coll Cardiol. 2009;53(11):919-28. 